(C2008 IEEE. Personal use of this material is permitted. However, permission to reprint/republish this material for advertising or promotional purposes or for creating new collective works for resale or redistribution to servers or lists, or to reuse any copyrighted component of this work in other works must be obtained from the IEEE 


\title{
Microarray Image Enhancement by Denoising Using Stationary Wavelet Transform
}

\author{
X. H. Wang, Robert S. H. Istepanian, Senior Member, IEEE, and Yong Hua Song, Senior Member, IEEE
}

\begin{abstract}
Microarray imaging is considered an important tool for large scale analysis of gene expression. The accuracy of the gene expression depends on the experiment itself and further image processing. It's well known that the noises introduced during the experiment will greatly affect the accuracy of the gene expression. How to eliminate the effect of the noise constitutes a challenging problem in microarray analysis. Traditionally, statistical methods are used to estimate the noises while the microarray images are being processed. In this paper, we present a new approach to deal with the noise inherent in the microarray image processing procedure. That is, to denoise the image noises before further image processing using stationary wavelet transform (SWT). The time invariant characteristic of SWT is particularly useful in image denoising. The testing result on sample microarray images has shown an enhanced image quality. The results also show that it has a superior performance than conventional discrete wavelet transform and widely used adaptive Wiener filter in this procedure.
\end{abstract}

Index Terms-Stationary wavelet transform, microarray images, denoising.

\section{INTRODUCTION}

$\mathbf{I}$ T IS WELL KNOWN that microarray imaging is a recent cutting-edge technology in bioinformatics which can monitor thousand of genes simultaneously. The origin of this imaging technique was evolved from E. Southern's technique in the 1970s [1] and sparked in the last decade by two key innovations. One was the use of nonporous solid support, such as glass, to facilitate miniaturization and fluorescent-based detection, which was pioneered by P. Brown [2]. Another was the development of methods, by S. Fodor and colleagues [3], for high-density spatial synthesis of oligonucleotide. After that, thousands of oligonucleotides and cDNAs could be globally viewed at the same time. This provides a systematic and comprehensive way to survey the DNA and RNA variations [4], which could become a standard tool for both molecular biology research and genomic clinical diagnosis, such as cancer diagnosis [5], [6], type 1 [7] and type 2 [8] diabetes diagnosis.

In general, the results of the microarray processing combine two sample images that after further image processing, gene ex-

\footnotetext{
Manuscript received December 16, 2002; revised June 16, 2003. Asterisk indicates corresponding author.

*X. H. Wang is with the Mobile Information and Network Technologies Centre, School of Computing and Information Systems, Kingston University, London Kingston Upon Thames KT1 2EE, U.K. (e-mail: xinheng.wang@kingston.ac.uk).

R. S. H. Istepanian is with Mobile Information and Network Technologies Centre, School of Computing and Information Systems, Kingston University London, Kingston Upon Thames KT1 2EE, U.K.

Y. H. Song is with the Department of Electronic and Computer Engineering,

Brunel University, Uxbridge UB8 3PH, U.K.

Digital Object Identifier 10.1109/TNB.2003.816225
}

pression data can be produced for further analysis, such as gene clustering or identification. These three crucial steps, experiment, image processing and data analysis, determine the success or not of the microarray analysis. Image processing plays a potentially large impact on the subsequent analysis. In recent years, large number of commercial tools have been developed in microarray image processing [9]-[15]. The tasks of all these tools mainly focus on two major targets, namely: spot segmentation and spot intensity extraction. However, the quality of the images from the experiments is not always perfect. The gene array experiments involve a large number of error-prone steps which lead to a high level of noise in the resulting images [16]. Hence, the accuracy of the gene expressions derived from these images will largely be affected in the process.

In order to assure the accuracy of the gene expression, normally the replicated experiments and incorporated statistical methods are needed to estimate the errors. Such statistical methods include analysis of variance introduced by Kerr [17], ratio distribution by Chen [18] and Ermolaeva [19], Gamma distribution by Newton [20], empirical Bayes model by Lonnstedt and Speed [21], and Bayesian Estimation of Array Measurements (BEAM) by Dror [22]. These methods deal mainly with measurement error, such as preparation of the sample, cross hybridization, and fluctuation of fluorescence value from gene to gene. But none deals particularly with the effect of the noise.

In this paper, we proposed a new approach based on wavelet theory to provide an enhanced approach for eliminating such noise source and ensure better gene expression. It is well known that wavelet transform is a signal processing technique which can display the signals on in both time and frequency domain. Wavelet transform is superior approach to other time-frequency analysis tools because its time scale width of the window can be stretched to match the original signal, especially in image processing studies. This makes it particularly useful for nonstationary signal analysis, such as noises and transients. For a discrete signal, a fast algorithm of discrete wavelet transform(DWT) is multiresolution analysis, which is a nonredundant decomposition [23]. The drawback of nonredundant transform is their noninvariance in time/space, i.e., the coefficients of a delayed signal are not a time-shifted version those of the original signal. The stationary wavelet transform (SWT) was introduced in 1996 to make the wavelet decomposition time invariant [24]. This improves the power of wavelet in signal de-noising. In this paper, we apply the SWT method to preprocess the microarray images for removing the random noises. We also compare this method with other two traditional denoising methods, namely DWT and Wiener 


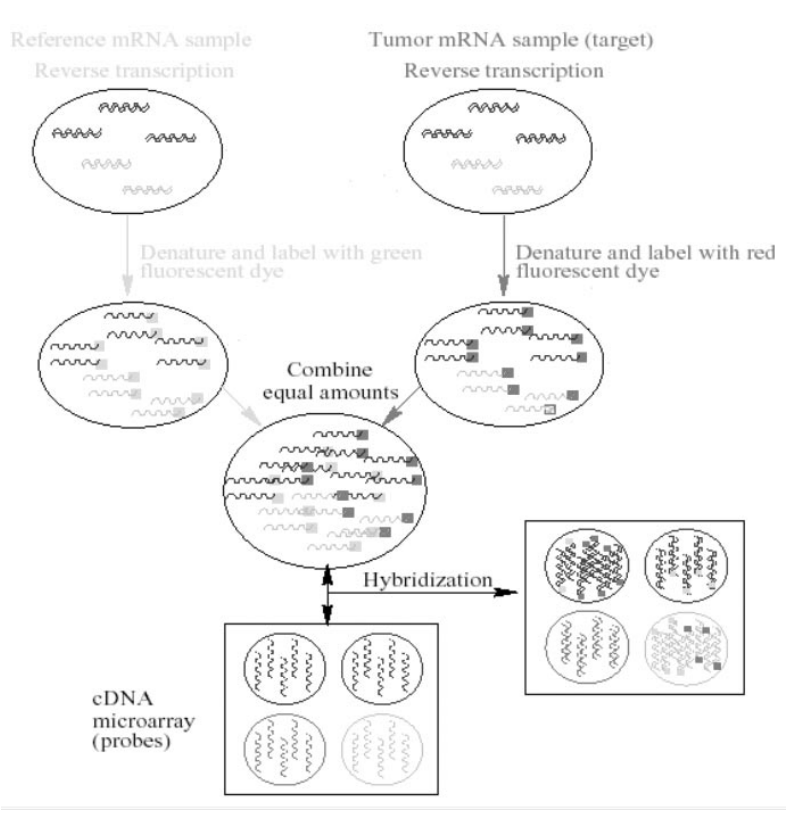

Fig. 1. Schematic diagram of microarray experiment.

filter, to validate the enhanced characteristics of this method to microarray image processing.

\section{MicRoARRAY IMAGING AND NOISE SOURCES}

It is well known microarray technology can monitor thousand of DNA sequences in a high density array on a glass. The basic procedure for a microarray experiment is simply described as follow. Two mRNA samples are reverse-transcribed into cDNA, labeled using different fluorescent dyes (e.g., the red fluorescent dye $\mathrm{Cy} 5$ and the green fluorescent dye Cy3), then mixed and hybridized with the arrayed DNA sequences. After this competitive hybridization, the slides are imaged using a scanner which makes fluorescence measurement for each dye. From the differential hybridization of the two samples, the relative abundance of the spotted DNA sequences can be assessed. A schematic diagram for this process created is shown in Fig. 1 [25].

The results of the microarray experiment are two 16-bit tagged mage files, one for each fluorescent dye. Fig. 2 shows one example of the microarray image.

As shown in Fig. 2, the image is not perfect and includes noisy sources that blur such images for further gene expression experimentation. The noise source originates from different sources during the course of experiment, such as photon noise, electronic noise, laser light reflection, dust on the slide, and so on. Hence, it is crucial to denoise the resultant image within this process.

Exciting methods to reduce the noise source include using clean glass slide and using a higher laser power rather than a higher PMT voltages. However, there are not adequate for the required image qualities and an enhanced software procedure embedded within the process in a much better alternative. In this paper, we focus on the implementation of the SWT method to the denoising on microarray images.

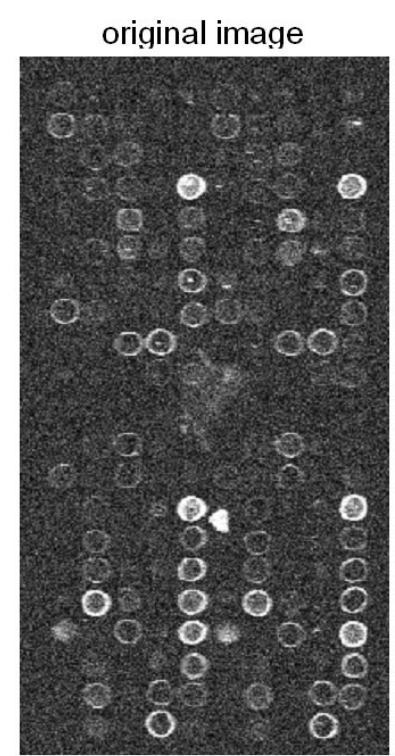

Fig. 2. Microarray image.

\section{StATIONARY WAVELET MEthoD}

In this section, we present the basic principals of the SWT method. In summary, the SWT method can be described as follows. At each level, when the high-pass and low-pass filters are applied to the data, the two new sequences have the same length as the original sequences. To do this, the original data is not decimated. However, the filters at each level are modified by padding them out with zeros.

Supposing a function $f(x)$ is projected at each step $j$ on the subset $V_{j}\left(\ldots \ldots \subset V_{3} \subset V_{2} \subset V_{1} \subset V_{0}\right)$. This projection is defined by the scalar product $c_{j, k}$ of $f(x)$ with the scaling function $\phi(x)$ which is dilated and translated

$$
\begin{aligned}
c_{j, k} & =\left\langle f(x), \phi_{j, k}(x)\right\rangle \\
\phi_{j, k}(x) & =2^{-j} \phi\left(2^{-j} x-k\right)
\end{aligned}
$$

where $\phi(x)$ is the scaling function, which is a low-pass filter. $c_{j, k}$ is also called a discrete approximation at the resolution $2^{j}$.

If $\varphi(x)$ is the wavelet function, the wavelet coefficients are obtained by

$$
\omega_{j, k}=\left\langle f(x), 2^{-j} \varphi\left(2^{-j} x-k\right)\right\rangle .
$$

$\omega_{j, k}$ is called the discrete detail signal at the resolution $2^{j}$.

As the scaling function $\phi(x)$ has the following property:

$$
\frac{1}{2} \phi\left(\frac{x}{2}\right)=\sum_{n} h(n) \phi(x-n) .
$$

$c_{j+1, k}$ can be obtained by direct computation from $c_{j, k}$

$$
\begin{aligned}
c_{j+1, k} & =\sum_{n} h(n-2 k) c_{j, n} \quad \text { and } \\
\frac{1}{2} \varphi\left(\frac{x}{2}\right) & =\sum_{n} g(n) \phi(x-n) .
\end{aligned}
$$


The scalar products $\left\langle f(x), 2^{-(j+1)} \varphi\left(2^{-(j+1)} x-k\right)\right\rangle$ are computed with

$$
\omega_{j+1, k}=\sum_{n} g(n-2 k) c_{j, n} .
$$

Equations (4) and (5) are the multiresolution algorithm of the traditional DWT. In this transform, a downsampling algorithm is used to perform the transformation. That is, one point out of two is kept during transformation. Therefore, the whole length of the function $f(x)$ will reduce by half after the transformation. This process continues until the length of the function becomes one.

However, for stationary or redundant transform, instead of downsampling, an upsampling procedure is carried out before performing filter convolution at each scale. The distance between samples increasing by a factor of two from scale $j$ to the next. $c_{j+1, k}$ is obtained by

$$
c_{j+1, k}=\sum_{l} h(l) c_{j, k+2^{j} l}
$$

and the discrete wavelet coefficients

$$
\omega_{j+1, k}=\sum_{l} g(l) c_{j, k+2^{j} l} .
$$

The redundancy of this transform facilitates the identification of salient features in a signal, especially for recognising the noises.

This is the transform for one-dimensional signal. For a twodimensional image (microarray image in this case), we separate the variables $x$ and $y$ and have the following wavelets.

- Vertical wavelet: $\varphi^{1}(x, y)=\phi(x) \varphi(y)$

- Horizontal wavelet: $\varphi^{2}(x, y)=\varphi(x) \phi(y)$

- Diagonal wavelet: $\varphi^{3}(x, y)=\varphi(x) \varphi(y)$.

Thus, the detail signal is contained in three subimages

$$
\begin{aligned}
& \omega_{j+1}^{1}\left(k_{x}, k_{y}\right)=\sum_{l_{x}=-\infty}^{+\infty} \sum_{l_{y}=-\infty}^{+\infty} g\left(l_{x}\right) h\left(l_{y}\right) c_{j, k+2^{j}}\left(l_{x}, l_{y}\right) \\
& \omega_{j+1}^{2}\left(k_{x}, k_{y}\right)=\sum_{l_{x}=-\infty}^{+\infty} \sum_{l_{y}=-\infty}^{+\infty} h\left(l_{x}\right) g\left(l_{y}\right) c_{j, k+2^{j}}\left(l_{x}, l_{y}\right) \\
& \omega_{j+1}^{3}\left(k_{x}, k_{y}\right)=\sum_{l_{x}=-\infty}^{+\infty} \sum_{l_{y}=-\infty}^{+\infty} g\left(l_{x}\right) g\left(l_{y}\right) c_{j, k+2^{j}}\left(l_{x}, l_{y}\right) .
\end{aligned}
$$

\section{IMPLEMENTATION OF SWT IN MicroarRAY IMAGE DE-NOISING AND RESULTS}

In this section, the implementation issues of the SWT method for enhanced microarray image quality are described. A comparative analysis of this method with conventional DWT and Wiener filter is presented to validate the results obtained.

The wavelet denoising is achieved via thresholding or shrinkage. The wavelet thresholding procedure removes noise by thresholding only the wavelet coefficient of the

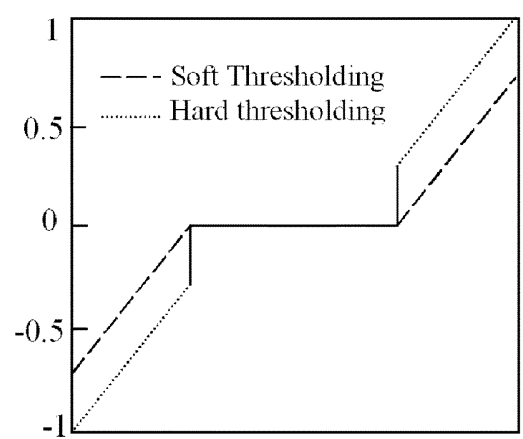

Fig. 3. Soft and hard thresholding.

detail subbands, while keeping the low resolution coefficients unaltered. There are two thresholding methods frequently used, soft-thresholding and hard-thresholding functions. The soft-thresholding rule is normally chosen over hard-thresholding in de-noising. There are several reasons for this. First, soft-thresholding has been shown to achieve near-optimal minimax rate. Second, the optimal soft-thresholding estimator yields a smaller risk thanks the optimal hard-thresholding estimator. Finally, in practice, the soft-thresholding method yields more visually enhanced images over hard-thresholding because the latter is discontinuous and yields abrupt artefacts in the recovered images, especially when the noise energy is significant.

The general soft-thresholding function is defined by

$$
\eta_{T}(x)=\operatorname{sgn}(x) \cdot \max (|x|-T, 0) .
$$

Its function is also illustrated in Fig. 3 and compared with the hard thresholding.

By the soft thresholding, the general denoising procedure involves three steps, including:

i) decompose;

ii) threshold detail coefficients;

iii) reconstruct.

In the three steps, a signal is first decomposed by the wavelet transform. Then the decomposition coefficients are thresholded by the thresholding rule. After the thresholding, a new coefficient is obtained. This new coefficient is then reconstructed to a new signal. This is filtered signal by wavelet transform.

In this paper, we apply the biorthogonal wavelet procedure to decompose the image using Matlab. After the decomposition procedure at two levels, three detailed subimages and one approximation image at each level are produced. The three detailed subimages in level one, after the decomposition procedure outlined above with resultant decomposed images, are shown in Fig. 4.

Next, the SureShrink thresholding algorithm is applied to the above three subimages [26]. The thresholding estimator for $j$ th level of decomposition with coefficient $\hat{d}$ is given as

$$
\begin{aligned}
& \operatorname{SURE}_{j}=2^{j} \sigma_{j}^{2}+\sum_{k=0}^{2^{j}-1} \min \left(\hat{d}_{j k}^{2}, \lambda_{j}^{2}\right) \\
&-2 \sigma_{j}^{2} I\left(\left|\hat{d}_{j k}\right| \leq \lambda_{j}\right)
\end{aligned}
$$


H Detail

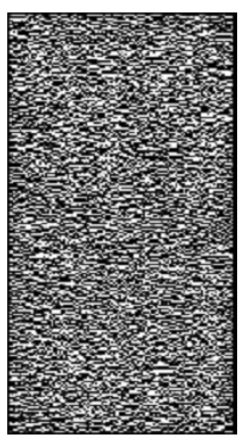

V. Detail

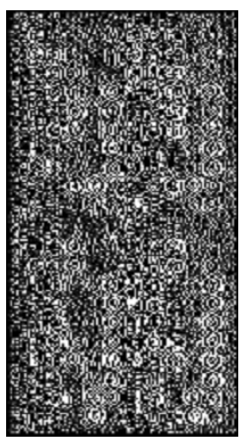

D. Detail

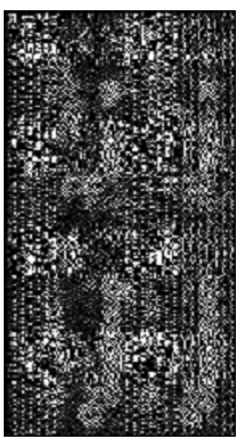

Fig. 4. Three-dimensional detail subimages.

where $\sigma$ is the variance, $\lambda$ is the thresholding value $\hat{\lambda}_{j}=\arg \min \operatorname{SURE}\left(\lambda_{j}\right)$. For the sparse case, the thresholding value is set as $\sigma \sqrt{2 \log (M)}$, where $M$ is the length of the signal.

Through the thresholding, the noise sources in detail images are eliminated at each level. After the denoising, the denoised detail images and the approximation image are reconstructed and the result is shown in Fig. 5(b). The above application of SWT via SureShrink thresholding is implemented in Matlab. For the comparison, the original image is also displayed in Fig. 5(a).

Traditionally, wavelet transform (DWT) is also performed following the above procedure using same wavelet, decomposition level, and thresholding function. The resultant denoised image using DWT is shown in Fig. 5(c).

In order to compare the performance of the SWT in microarray image denoising with other methods, the widely used adaptive filter in denoising, Wiener filter, is also applied. The result of Wiener filtering is shown Fig. 5(d).

In order to provide a quantitative measure of the resultant images, the universal index proposed in [27] is presented here. This is defined as

$$
Q=\frac{4 \sigma_{x y} \bar{x} \cdot \bar{y}}{\left(\sigma_{x}^{2}+\sigma_{y}^{2}\right)\left[(\bar{x})^{2}+(\bar{y})^{2}\right]}
$$

where $\bar{x}=(1 / N) \sum_{i=1}^{N} x_{i}, \bar{y}=(1 / N) \sum_{i=1}^{N} y_{i}$

$$
\begin{aligned}
\sigma_{x}^{2} & =\frac{1}{N-1} \sum_{i=1}^{N}\left(x_{i}-\bar{x}\right)^{2} \\
\sigma_{y}^{2} & =\frac{1}{N-1} \sum_{i=1}^{N}\left(y_{i}-\bar{y}\right)^{2} \\
\sigma_{x y} & =\frac{1}{N-1} \sum_{i=1}^{N}\left(x_{i}-\bar{x}\right)\left(y_{i}-\bar{y}\right) .
\end{aligned}
$$

Using this index, the quality of the denoised images by SWT, DWT, and Wiener filter are $0.7927,0.7905$, and 0.6842, respectively. The SWT achieves $16 \%$ better performance than Wiener filter. Extensive tests are also performed using various wavelets for the same image. The denoised image quality via several example wavelets, and Wiener filter performance are shown in Table I. It is clear that the SWT denoising achieves a better image quality than DWT for every wavelet if the decomposition

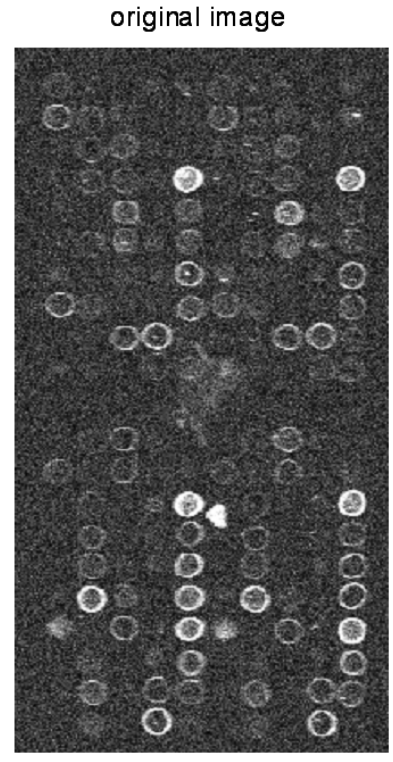

(a)

denoised signal

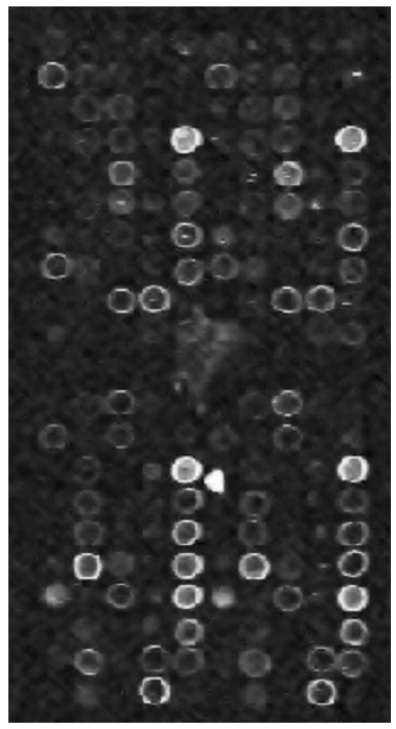

(c)

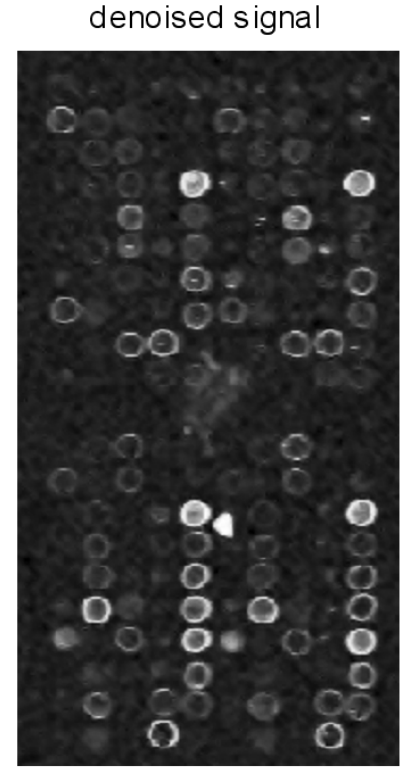

(b)

wiener filtered

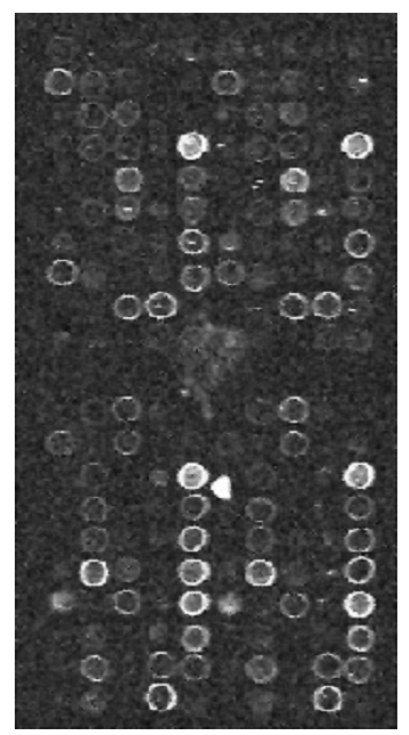

(d)
Fig. 5. Original and the denoised images.

TABLE I

COMPARATIVE PERFormance OF THE QUALITy INDEXES USING SWT, DWT, AND WIENER FILTER

\begin{tabular}{c|c|c|c}
\hline Wavelet & SWT & DWT & $\begin{array}{c}\text { Wiener } \\
\text { Filter }\end{array}$ \\
\hline Db2 & 0.7903 & 0.7846 & 0.6842 \\
\hline Haar & 0.7928 & 0.7883 & 0.6842 \\
\hline Bior1.3 & 0.7927 & 0.7905 & 0.6842 \\
\hline Bior1.5 & 0.7926 & 0.7904 & 0.6842 \\
\hline Coif1 & 0.7893 & 0.7856 & 0.6842 \\
\hline Sym2 & 0.7903 & 0.7846 & 0.6842 \\
\hline
\end{tabular}

level and thresholding rules keep the same. For Wiener filter, the performance is far poorer than that of the SWT.

In order to test the effectiveness of the SWT algorithm and compare it with the other two algorithms, we apply the three algorithms on another sample image [Fig. 6(a)]. A repeated pro- 
original image

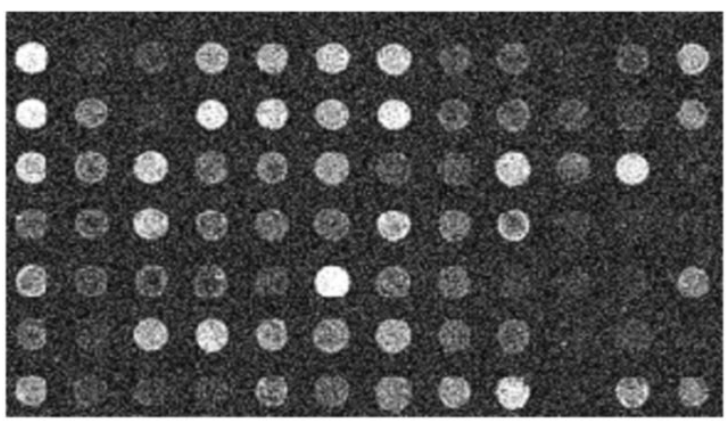

(a)

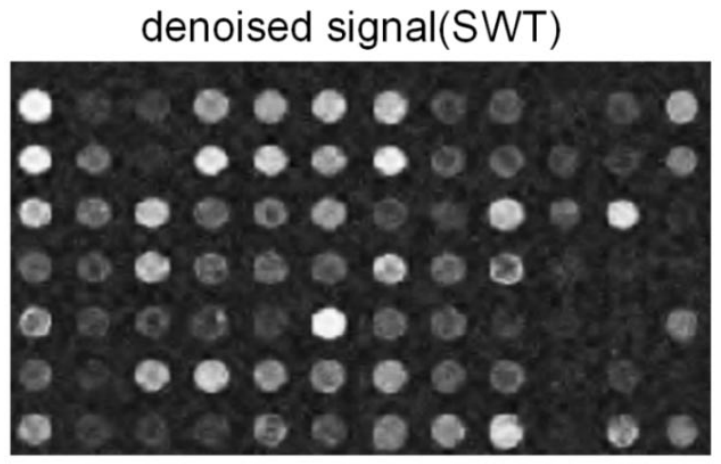

(b)

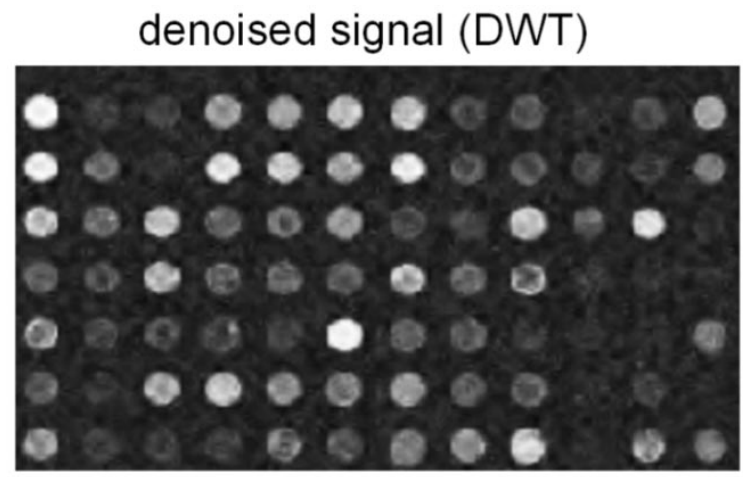

(c)

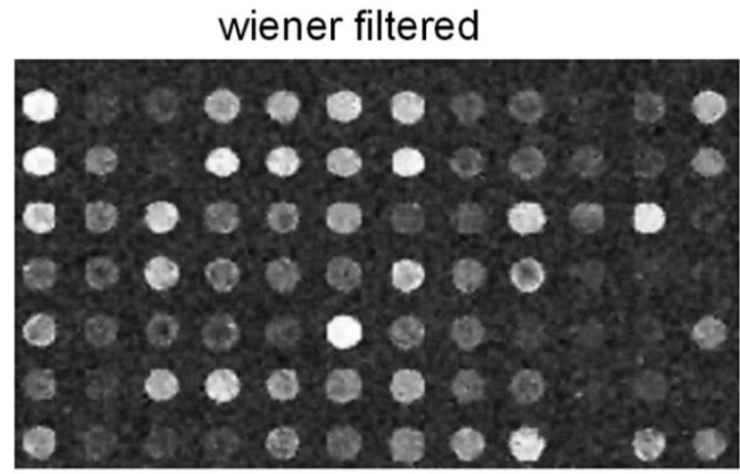

(d)

Fig. 6. Example 2 of original and filtered microarray images.

cedure of the algorithms is applied on this image. The denoised images are shown in Fig. 6(b)-(d) for SWT denoising, DWT denoising, and Wiener filtering, respectively. Its corresponding quality indexes for the three algorithms are $0.8323,0.8243$, and
0.7426 , respectively. Overall, the same consistent characteristic is obtained.

\section{CONCLUSION}

In this paper, a new wavelet approach to deal with microarray denoising image analysis is presented. The new approach adopts the denoising by SWT. This image processing method has an obvious advantage, namely time invariance. This makes it particularly useful in recognising the noises in microarray images. The simulation results applied on microarray image examples verified this enhanced characteristic and denoising quality of the image analysis. The SWT provides a better performance in denoising microarray image than traditional wavelet transform method.

The results also show that the stationary wavelet denoising has a $16 \%$ better performance than Wiener filter which is widely used in commercial denoising software system. The application of this method would improve the accuracy of gene expression, and therefore easily identify the diseased gene for diagnosing critical diseases.

\section{REFERENCES}

[1] E. M. Southern, "Detection of specific sequences among DNA fragments separated by gel electrophoresis," J. Mol. Biol., vol. 98, pp. 503-517, 1975.

[2] M. Schena, D. Shalon, R. W. Davis, and P. O. Brown, "Quantitative monitoring of gene expression patterns with a complementary DNA microarray," Science, vol. 270, pp. 467-470, 1995.

[3] S. P. A. Fodor, J. L. Read, M. C. Pirrung, L. Stryer, A. T. Lu, and D. Solas, "Light-directed, spatially addressable parallel chemical synthesis," Science, vol. 251, pp. 767-773, 1991.

[4] E. S. Lander, "Array of hope,” Nature Genetics Suppl., vol. 21, pp. 3-4, Jan. 1999.

[5] A. Alizadeh, M. Eisen, P. O. Brown, and L. M. Staudt, "Probing lymphocyte biology by genomic-scale gene expression analysis," J. Clin. Immunol., vol. 18, pp. 373-379, 1998.

[6] C. M. Perou, S. S. Jeffrey, M. Van de Rijn, C. A. Rees, M. B. Eisen, D. T. Ross, A. Pergamenschikov, C. F. Williams, S. X. Zhu, J. C. F. Lee, D. Lashkari, D. Shalon, P. O. Brown, and D. Botstein, "Distinctive gene expression patterns in human mammary epithelial cells and breast cancers," Proc. Nat. Acad. Sci., vol. 96, pp. 9212-9217, 1999.

[7] L. M. Scearce, J. E. Brestelli, S. K. McWeeney, C. S. Lee, J. P. Mazzarelli, F. Deborah, A. S. Pizarro, C. J. Stoechert Jr., S. P. Sandra, M. A. Permutt, J. Brown, A. Douglas, and K. H. Kasestner, "Functional genomics of the endocrine pancreas: The pancreas clone set and pancchip, new resources for diabetes research," Diabetes, vol. 51, pp. 1997-2004, 2002 .

[8] S. T. Nadler, J. P. Stoehr, K. L. Schueler, G. Tanimoto, B. S. Yandel, and A. D. Attie, "The expression of adipogenic genes is decreased in obesity and diabetes mellitus," Proc. Nat. Acad. Sci., vol. 97, pp. 11371-11376, 2000.

[9] T. Nagashima, K. Takahashi, H. Bono, Y. Okazaki, and A. Konagaya, "Fully-automated spot recognition and quantification from cDNA microarray images," in Proc. Int. Conf. Parallel and Distributed Processing Techniques and Applications (PDPTA'2001), vol. 3, pp. 1291-1297.

[10] M. Steinfath, W. Wruch, H. Seidel, H. Lehrach, U. Radelof, and J. O'Brien, "Automated image analysis for array hybridization experiments," Bioinformatics, vol. 17, no. 7, pp. 634-641, 2001.

[11] D. Bozinov and J. Rahnenfuhrer, "Unsupervised technique for robust target separation and analysis of DNA microarray spots," Bioinformatics, vol. 18, no. 5, pp. 747-756, 2002.

[12] W. Wruch, H. Griffiths, M. Steinfath, H. Lehrach, U. Radelof, and J. O'Brien, "Xdigitise: Visualization of hybridization experiments," Bioinformatics, vol. 18, no. 5, pp. 757-760, 2002.

[13] M. A. Zapala, D. J. Lockhart, D. G. Pankratz, A. J. Garcia, C. Barlow, and D. J. Lockhard, "Software and methods for oligonucleotide and cDNA array data analysis," Genome Biol., vol. 3, no. 6, 2002. 
[14] A. N. Jain, T. A. Tokuyasu, A. M. Snijders, R. Segraves, D. G. Albertson, and D. Pinkel, "Fully automatic quantification of microarray image data," Genome Res., vol. 12, pp. 325-332, 2002.

[15] X. Wang, S. Ghosh, and S. W. Guo, "Quantitative quality control in microarray image processing and data acquisition," Nucleic Acids Res., vol. 29 , no. 15,2001 . e75.

[16] J. Schuchhardt, D. Beule, A. Malik, E. Wolski, H. Eickhoff, H. Lehrach, and H. Herzel, "Normalization strategies for cDNA microarrays," $\mathrm{Nu}$ cleic Acids Res., vol. 28, no. 10, 2000. E47.

[17] M. K. Kerr, M. Martin, and G. A. Churchill, "Analysis of variance for gene expression microarray data," J. Comput. Biol., vol. 7, pp. 819-837, 2001.

[18] Y. Chen, E. R. Dougherty, and M. L. Bittner, "Ratio-based decision and the quantitative analysis of cDNA microarray images," J. Biomed. Optics., pp. 364-374, 1997.

[19] O. Ermolaeva, M. Rastogi, K. D. Pruitt, G. D. Schuler, M. L. Bittner, Y Chen, R. Simon, P. Meltzer, J. M. Trent, and M. Boguski, "Data management and analysis for gene expression arrays," Nature Genetics, vol. 20, pp. 19-23, 1998.

[20] M. A. Newton, C. M. Kendziorski, C. S. Richmond, F. R. Blattner, and K. W. Tsui, "On differential variability of expression ratios: Improving statistical inference about gene expression changes from microarray data," J. Computat. Biol., vol. 8, pp. 37-52, 2001.

[21] I. Lonnstedt and T. Speed, "Replicated microarray data," Statistica Sinica, vol. 12, pp. 31-46, 2002.

[22] R. Dror, J. Murnick, and N. Rinaldi, "A bayesian approach to transcript estimation from gene array data: The BEAM technique," presented at the Proc. 6th Annu. Int. . Conf. Research in Computational Molecular Briology, Washington, DC, Apr. 2002.

[23] S. Mallat, "A theory for multiresolution signal decomposition: The wavelet representation," IEEE Trans. Pattern Anal. Machine Intell., vol. 11, pp. 674-693, July 1989.

[24] J. C. Pesquet, H. Krim, and H. Carfantan, "Time-invariant orthonorma wavelet representations," IEEE Trans. Signal Processing, vol. 44, pp. 1964-1970, Aug. 1996.

[25] Y. H. Yang, M. J. Buckley, S. Dudoit, and T. P. Speed, "Comparison of methods for image analysis on cDNA microarray data,", Tech. Rep. 584, 2000.

[26] D. L. Donoho and I. M. Johnstone, "Adapting to unknown smoothness via wavelet shrinkage," J. Amer. Statist. Assoc., vol. 90, no. 432, pp. 1200-1224, Dec. 1995

[27] Z. Wang and A. Bovik, "A universal image quality index," IEEE Trans. Signal Processing Lett., vol. 9, pp. 81-84, Mar. 2002

X. H. Wang, photograph and biography not available at the time of publication.

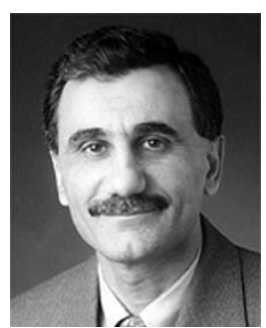

Robert S. H. Istepanian (M'91-SM'97) received the Ph.D. degree in electronic and electrical engineering from Loughborough University, Loughborough, U.K., in 1994.

From 1984 to 1998, he worked in different overseas industrial and academic positions. In 1988, he was a Visiting Research Fellow in the Department of Electronic and Electrical Engineering, Loughborough University. From 1994 to 1995, he was with the same department as a Postdoctoral Research Fellow. From 1996 to 1999, he was a Senior Lecturer at the University of Portsmouth, Portsmouth, U.K. From 1999 to 2000, he was an Associate Professor at Ryerson University, Toronto, ON, Canada, and also an Adjunct Professor with the University of Western Ontario, London, ON, Canada. He was also the Head of the Mobile Information Engineering and E-Med Systems research group in the Department of Electronic and Computer Engineering, Brunel University, London, U.K. He is currently Professor of Data Communications in the School of Computing and Information Systems and Director of the Mobile Information and Network Technologies Research Centre (MINT), Kingston University, London, U.K. He is Recipient, Investigator, and Coinvestigator of research grants from the Engineering and Physical Science Research Council, British Council, and Royal Society on intelligent computational systems applied to telesurgery systems. He is also a lead partner on several EU-funded projects on e-health. Other collaborative and funded projects from industry (Vodafone, Orange, and Motorola UK) in the areas of m-health systems and microarray imaging technologies are also ongoing. He has published more than 120 refereed journal and conference papers, mostly in the areas of of wireless telemedicine and biomedical signal processing, and has edited three books in the areas of m-health, biomedical communications, and signal processing. His current research lies in the cognate areas of wireless communications and e-health and advanced coding theory for genomics and microarray imaging technologies.

Dr. Istepanian currently serves on the advisory board of IEEE TRANSACTIONS ON INFORMATION TECHNOLOGY IN BIOMEDICINE, and was one of the Founding Special Area Editors on mobile telemedicine. He was also the Guest Editor of the IEEE TRANSACTIONS ON INFORMATION TECHNOLOGY IN BIOMEDICINE Special Issue on Mobile Telemedicine Systems (Sept. 2000), and is Guest Editor of the forthcoming Special Issue on m-Health to be published in late 2003. He was the Co-Chair of the UK/RI chapter of the IEEE Engineering in Medicine and Biology Society (EMBS). He has also served on numerous technical committees, and has chaired and was an invited speaker for several national and international IEEE conferences, including the Telemed conferences of the Royal Society of Medicine, the Annual International Conference of the EMBS (EMBS' 98 and '99), and the 2000 World Medical Congress. He was also the Technical Co-Chair of the 2003 IEEE EMBS Conference on Information Technology and Applications in Biomedicine (ITAB) and the Fifth International Workshop on Enterprise Networking and Computing in HealthCare (HealthCom'03). His biography is included in Who's Who in Science and Engineering (New Providence, NJ: Marquis, 2003).

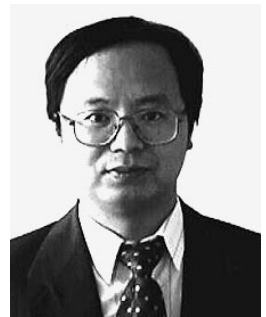

Yong Hua Song (M'90-SM'94) is currently a Professor of Network Systems at Brunel University, London, U.K., where he is Director of Brunel Network Systems Research Centre. His research interests include applications of advanced intelligent techniques and signal processing methods in various engineering problems. 